\title{
Cognitive Correlates of Hippocampal Atrophy and Ventricular Enlargement in Adults with or without Mild Cognitive Impairment
}

\author{
Naira Goukasian ${ }^{a}$ Shai Porat $^{\mathrm{b}}$ Anna Blanken ${ }^{\mathrm{b}}$ David Avila ${ }^{c}$ \\ Dimitar Zlatev $^{d}$ Sona Hurtz ${ }^{\mathrm{e}}$ Kristy S. Hwang ${ }^{f}$ Jonathan Pierce $^{g}$ \\ Shantanu H. Joshig Ellen Woog Liana G. Apostolovah-j \\ a University of Vermont, Larner College of Medicine, Burlington, VT, USA; ${ }^{b}$ Department \\ of Psychology, University of Southern California, Los Angeles, CA, USA; ' $I$ rvine School \\ of Medicine, University of California, Irvine, CA, USA; d Massachusetts General Hospital, \\ Boston, MA, USA; ${ }^{e}$ Drexel University College of Medicine, Philadelphia, PA, USA; ${ }^{f}$ Emory \\ University School of Medicine, Atlanta, GA, USA; ${ }^{9}$ Department of Neurology, University of \\ California, Los Angeles, CA, USA; ${ }^{\text {h}}$ Department of Neurology, Indiana University School of \\ Medicine, Indianapolis, IN, USA; 'Department of Radiology and Imaging Sciences, Indiana \\ University School of Medicine, Indianapolis, IN, USA; jDepartment of Medical and Molecular \\ Genetics, Indiana University School of Medicine, Indianapolis, IN, USA
}

\section{Keywords}

Alzheimer's disease - Mild cognitive impairment · Imaging · MRI · Hippocampal atrophy ·

Ventricular enlargement

\section{Abstract}

We analyzed structural magnetic resonance imaging data from 58 cognitively normal and 101 mild cognitive impairment subjects. We used a general linear regression model to study the association between cognitive performance with hippocampal atrophy and ventricular enlargement using the radial distance method. Bilateral hippocampal atrophy was associated with baseline and longitudinal memory performance. Left hippocampal atrophy predicted longitudinal decline in visuospatial function. The multidomain ventricular analysis did not reveal any significant predictors.

\section{Introduction}

According to the 2010 United States census, it is estimated that 5 million Americans currently suffer from Alzheimer's disease (AD); a number that is projected to triple by 2050 $[1,2]$. Neuropsychological testing is an essential tool for assessing cognitive function in both 
the prodromal and dementia stages [3] and can reveal areas of cognitive decline relatively early in the disease course [4]. Mild cognitive impairment (MCI) is the intermediate stage between the cognitively normal (NC) and the dementia state. MCI patients manifest cognitive difficulties greater than expected for age, but can still live independently [3-5]. An estimated $60 \%$ of people living with MCI progress to dementia within 5 years $[5,6]$.

$\mathrm{MCI}$ and $\mathrm{AD}$ patients exhibit severe atrophy in hippocampal regions when compared to healthy controls [7-10]. Hippocampal atrophy predates clinical symptoms and has proven to be a strong predictor of future cognitive decline $[11,12]$. Hippocampal atrophy is associated with neurofibrillary tangle deposition and neuronal loss [13, 14].

Ventriculomegaly is commonly observed in both MCI and AD patients $[10,15]$. Ventriculomegaly is thought to result from neuronal loss with subsequent axonal degeneration, leading to ex vacuo ventricular enlargement [10,16-21]. AD patients display up to $60 \%$ larger ventricles than those of healthy controls.

Neuropsychological testing performance has been previously associated with both hippocampal and ventricular volumetric change in AD [8, 15, 22-33]. Hippocampal atrophy and ventricular enlargement may help predict which healthy individual will decline into amnestic MCI $[8,11,12,15,34]$. Memory dysfunction, the salient feature of medial temporal lobe dysfunction, has been associated with hippocampal atrophy in AD [28-33]. Decline in language and executive function have also been associated with smaller hippocampi [8, 26-28]. Ventricular enlargement has been associated with worse performance on global cognitive tests, such as the MMSE and ADAScog $[15,22,24,25]$, and with executive decline [22].

The present study aims to further characterize the cognitive correlates of hippocampal atrophy and ventricular enlargement. Given that MCI patients often present with impairments in multiple cognitive domains, we wanted to examine whether these cognitive domains show an independent association with these neuroimaging biomarkers. We anticipated that in a multivariate model, hippocampal atrophy would most strongly associate with memory performance at baseline and with memory decline over time. We also hypothesized that ventriculomegaly will correlate better with non-memory domains, such as visuospatial and executive function.

\section{Methods}

\section{Subjects}

We enrolled 159 subjects who met the inclusion/exclusion criteria of the Imaging and Genetic Biomarkers of Alzheimer's Disease (ImaGene) Study at the University of California, Los Angeles (UCLA). Of those, 58 subjects were NC and 101 met criteria for MCI. ImaGene subjects were identified and recruited from two sources: (1) referrals from both UCLA and outside neurologists and (2) the UCLA Alzheimer's Disease Research Center (ADRC) ongoing longitudinal database study. The latter group consists of existing research participants who agreed to be contacted for future research opportunities and met ImaGene inclusion/ exclusion criteria. Informed consent was administered and obtained according to the guidelines of the UCLA Institutional Review Board.

To be included, subjects had to be at least 50 years old and able to independently carry out daily activities of living. The diagnostic evaluation consisted of a physician interview, general and neurological examination, as well as detailed neuropsychological evaluation. Initial and longitudinal diagnoses were determined by consensus decision by a group of neurologists and neuropsychologists using the National Institute of Neurologic and Communicative Disorders and Stroke and the Alzheimer's Disease and Related Disorders (NINCDS- 
Goukasian et al.: Cognitive Correlates of Hippocampal Atrophy and Ventricular Enlargement

ADRDA) criteria for AD [35] and Petersen criteria for MCI [6]. NC were required to fall within age-adjusted expectations on a detailed neuropsychological battery of tests and have a global clinical dementia rating (CDR) of 0 . MCI subjects were required to score $\geq 24$ on the MiniMental State Examination (MMSE) [36], and have objective cognitive decline at 1.5 SD or below age- and education-adjusted neuropsychological norms, a global CDR score $<1$, and intact activities of daily living. English language fluency and visual/hearing abilities sufficient for detailed neuropsychological testing were required for participation. Exclusionary criteria for both groups included concurrent medical problems of sufficient severity to impact cognition, history of alcohol or drug abuse in the past 2 years, age younger than 50 years, concurrent neurological or psychiatric illnesses, contraindications to MRI, cortical strokes or significant white matter changes, and visual and hearing impairment that could interfere with cognitive testing.

\section{Neuropsychological Data}

Subjects were tested annually with a neuropsychological battery consisting of the MMSE [36], Wechsler Test of Adult Reading (WTAR) [37], Wechsler Adult Intelligence Scale, 3rd edition (WAIS-III) [38] subtests, Wechsler Memory Scale, 3rd edition (WMS-III) subtests [39], Trailmaking A and B [40], Stroop Color-Word Interference Test [41], Boston Naming Test [42], Controlled Oral Word Association Test [43], Rey Osterrieth Complex Figure (ROCF) copy and 3-minute recall [44], California Verbal Learning Test, 2nd edition (CVLT-II) [45], and Wisconsin Card Sorting Test-64 (WCST-64) [46].

The neuropsychological data reduction technique has been previously described [47]. Briefly, we averaged the age-adjusted scores for all tests in five domains - memory, attention, executive, language, and visuospatial. Table 1 outlines the tests in each of the five cognitive domains. Cognitive rates of decline for each cognitive domain were calculated by taking the difference between the first $\left(S_{1}\right)$ and the last measurement $\left(S_{2}\right)$ scores and then dividing by the time between follow-up visits (FU): [( $\left.\left.\mathrm{S}_{1}-\mathrm{S}_{2}\right) / \mathrm{FU}\right]$.

\section{Imaging Acquisition}

Baseline 3D T1-weighted structural magnetic resonance scans were obtained from all ImaGene subjects according to the following protocol coronal Fast Low Angle Shot (FLASH) 3D T1 Magnetization Prepared Rapid Acquisition Gradient Echo (MPRAGE) with repetition time (TR) $28 \mathrm{~ms}$, echo time (TE) $4.5 \mathrm{~ms}$, FOV $22 \mathrm{~cm}$, matrix $256 \times 192$, slice/gap 1.5/0 mm. Subjects with motion or scanner artifacts were routinely rescanned, resulting in $100 \%$ usable baseline MRI data. T2 and fluid-attenuated inversion recovery (FLAIR) sequences were carefully inspected to exclude subjects with strokes, significant white matter changes, or structural lesions.

All MRI scans were aligned and scaled to the International Consortium for Brain Mapping ICBM53 average brain template [48] with a 9-parameter linear transformation (3 translations, 3 rotations, 3 scales) [49] and corrected for image non-uniformities using a regularized tricubic B-spline approach [50, 51].

\section{Hippocampal Analyses}

One experienced hippocampal tracer (LGA) manually traced the hippocampal formations of 20 randomly selected subjects (10 NC and $10 \mathrm{MCI}$ ) following the European AD ConsortiumAlzheimer's Disease Neuroimaging Initiative (EADC-ADNI) Harmonized Protocol for Manual Hippocampal Segmentation [52-54] (intra-rater reliability: intra-class correlation coefficient ICC $=0.97$ for both the left and right hippocampi; inter-rater reliability: ICC $=0.96$ for the left and ICC $=0.97$ for the right hippocampus [53]), while blinded to the subject's demographics and diagnosis. The hippocampus proper, dentate gyrus, alveus, and subiculum were included 
Table 1. Neuropsychological data groupings of the 5 main cognitive domains

\begin{tabular}{ll}
\hline Cognitive domain & Neuropsychological tests \\
\hline Attention & Digit Symbol, Digit Span forward and backward, Trails A, Stroop Color/Word \\
Memory & Logical Memory I and II, Visual Reproduction I and II, Trails 1-5, CVLT II - Short and \\
& Long Delay Free Recall, ROCF with 3-min delay \\
Language & Boston Naming Test, Animal Fluency, FAS Fluency \\
Visuospatial & ROCF Copy, Block Design \\
Executive & WCST Categories, Trails B, Stroop Interference \\
\hline
\end{tabular}

in the traces, as previously described [52]. The hippocampi were traced on contiguous coronal slices following the EADC-ADNI protocol with high intra- and inter-rater reliability [52, 54]. Anatomic landmarks were resolved in all three orthogonal viewing planes using interactive segmentation software prior to tracing. Standard neuroanatomical atlases were consulted as necessary for ambiguous boundaries $[55,56]$.

Hippocampal segmentation of the full sample was conducted with AdaBoost, which is a well-established automated machine-learning hippocampal segmentation algorithm [57$61]$. This technique has been previously described in detail $[60,61]$. Briefly, AdaBoost utilizes approximately 13,000 local features, such as image gradients, local curvature of image interfaces, and tissue classification as gray or white matter. AdaBoost also utilizes statistical information on the likely stereotaxic position of the hippocampus and develops segmentation rules based on the optimal combination of features. In the training phase, the algorithm applies mathematical approaches from the fields of machine learning and computer vision to estimate the optimal weighing of these features in a mathematical formula that computes the probability of any given voxel inside the hippocampus. The algorithm is tested in a training set and then applied to the full sample once the statistical rules for hippocampal segmentation are developed. The AdaBoost contours were made spatially uniform by modeling them as 3D parametric surface meshes [10]. All AdaBoost segmentations were visually inspected to make sure they appropriately captured the hippocampal anatomy.

\section{Ventricular Tracing and Extraction}

An expert rater (D.Z., intra-rater reliability Cronbach's alpha $=0.995)$ traced the lateral ventricles of 4 subjects in three partitions - frontal horn, temporal horn, and body/occipital horn, as previously described [62]. Traces were converted to one of four atlases, or 3D parametric ventricular mesh models. Atlases were then fluidly registered to each unsegmented study image [62]. Four separate ventricular segmentations for each participant were created and then averaged to reduce segmentation bias that occurs when a single atlas is used; reducing these errors allows true ventricle anatomy to be captured more accurately at the individual level.

\section{Radial Distance}

Once the segmented hippocampi and ventricular 3D parametric mesh models were created, we computed the medical core and radial distance from the medial core to each surface point for each subject. Calculating the distance from each surface point to the medial core of each individual hippocampus assessed the radial distance measurement [10]. Local expansions or contractions in corresponding surface morphology areas can thus be compared statistically between groups and over time, as previously demonstrated $[11,61$, 63-65]. 
Goukasian et al.: Cognitive Correlates of Hippocampal Atrophy and Ventricular Enlargement

Table 2. Baseline demographics

\begin{tabular}{|c|c|c|c|}
\hline Variable & $\begin{array}{l}\text { Normal } \\
\text { controls } \\
(n=58)\end{array}$ & $\begin{array}{l}\text { Mild cognitive } \\
\text { impairment } \\
(n=101)\end{array}$ & $p$ value \\
\hline Age (SD), years & $69.2(8.4)$ & $70.4(8.6)$ & 0.64 \\
\hline Education (SD), years & $17.5(2.0)$ & $15.8(2.8)$ & 0.01 \\
\hline \multicolumn{4}{|l|}{ Sex, $n(\%)$} \\
\hline Male & $32(55)$ & $44(44)$ & 0.19 \\
\hline Female & $26(45)$ & $57(56)$ & \\
\hline MMSE (SD) & $28.8(1.3)$ & $27.2(2.4)$ & $<0.001$ \\
\hline \multicolumn{4}{|l|}{ APOE $4, n(\%)$} \\
\hline 0 & $35(60)$ & $52(55)$ & 0.27 \\
\hline 1 & $21(36)$ & $39(38)$ & \\
\hline 2 & $2(4)$ & $10(7)$ & \\
\hline Memory domain, mean $Z$-score (SD) & $0.56(0.49)$ & $-0.49(0.69)$ & $<0.001$ \\
\hline Executive domain, mean $Z$-score (SD) & $0.49(0.39)$ & $-0.41(0.86)$ & $<0.001$ \\
\hline Attention domain, mean Z-score (SD) & $0.35(0.48)$ & $-0.26(0.67)$ & $<0.001$ \\
\hline Language domain, mean $Z$-score (SD) & $0.44(0.52)$ & $-0.37(0.74)$ & $<0.001$ \\
\hline Visuospatial domain, mean Z-score (SD) & $0.54(0.53)$ & $-0.29(0.75)$ & $<0.001$ \\
\hline
\end{tabular}

Table 3. Demographics of the longitudinal cohort

\begin{tabular}{|c|c|c|c|}
\hline Variable & $\begin{array}{l}\text { Normal } \\
\text { controls } \\
(n=58)\end{array}$ & $\begin{array}{l}\text { Mild cognitive } \\
\text { impairment } \\
(n=88)\end{array}$ & $p$ value \\
\hline Age (SD), years & $69.2(8.4)$ & $70.7(8.8)$ & 0.53 \\
\hline Education (SD), years & $17.5(2.0)$ & $15.6(2.8)$ & 0.02 \\
\hline \multicolumn{4}{|l|}{ Sex, $n(\%)$} \\
\hline Male & $32(55)$ & $40(45)$ & \multirow[t]{2}{*}{0.31} \\
\hline Female & $26(45)$ & $48(55)$ & \\
\hline Length of follow-up (SD), years & $4.3(1.4)$ & $3.1(1.3)$ & 0.91 \\
\hline MMSE (SD) & $28.8(1.3)$ & $27.2(2.4)$ & $<0.001$ \\
\hline \multicolumn{4}{|l|}{$\mathrm{APOE}, n(\%)$} \\
\hline 0 & $35(60)$ & $46(52)$ & \multirow[t]{3}{*}{0.35} \\
\hline 1 & $21(36)$ & $34(39)$ & \\
\hline 2 & $2(4)$ & $8(9)$ & \\
\hline Memory domain change, mean $Z$-score (SD) & $0.01(0.16)$ & $-0.05(0.19)$ & 0.065 \\
\hline Executive domain change, mean $Z$-score (SD) & $-0.08(0.28)$ & $-0.06(0.38)$ & 0.72 \\
\hline Attention domain change, mean $Z$-score (SD) & $-0.04(0.1)$ & $-0.09(0.38)$ & 0.195 \\
\hline Language domain change, mean $Z$-score (SD) & $0.02(0.19)$ & $-0.07(0.3)$ & 0.028 \\
\hline Visuospatial domain change, mean $Z$-score (SD) & $-0.04(0.24)$ & $-0.18(0.46)$ & 0.02 \\
\hline
\end{tabular}

\section{Statistical Analysis}

We conducted a two-tailed Student's $t$ test to compare continuous variables and a $\chi^{2}$ test was used to compare categorical variables. We then examined the associations between baseline performance and cognitive rates of change in each cognitive domain with ventricular and hippocampal volume. A two-step approach for each of the analyses was utilized. We first tested the association for each individual domain with hippocampal and ventricular radial distance, respectively, while correcting for age (unidomain approach). Next, we entered all significantly associated cognitive domain variables in the multidomain model and examined 


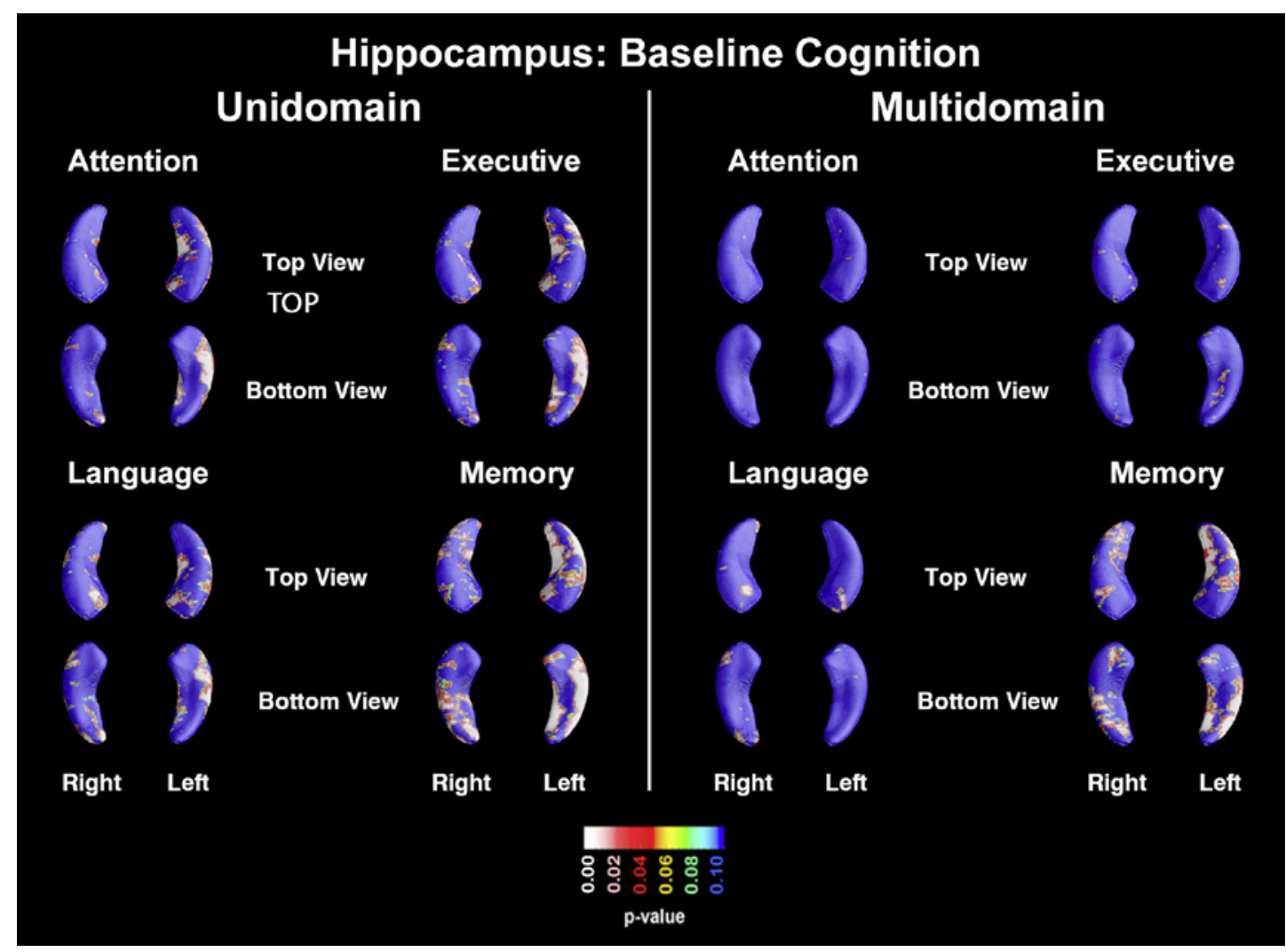

Fig. 1. Unidomain and multidomain maps showing the association of baseline cognitive performance and hippocampal radial distance.

the associations of each of these domains while correcting for all other domains that showed significance in the unidomain analysis, in addition to age (multidomain approach). Our 3D statistical maps were corrected for multiple comparisons using a permutation threshold of $p$ $<0.01$. We applied 100,000 permutations for each 3D statistical map to arrive at a single overall corrected $p$ value.

\section{Results}

As predicted, the normal control subjects performed significantly better on MMSE compared to the MCI group both at baseline and in follow-up ( $p<0.001$ for both). The normal controls were also significantly more educated compared to the MCI group both at baseline $(p=0.01)$ and in follow-up ( $p=0.02)$; however, since education did not show a significant effect on our primary outcome measures - cortical thickness and hippocampal atrophy - we did not explicitly control for education in our analyses. No other demographic differences were seen between the two groups (Tables 2, 3).

\section{Hippocampal Results}

Baseline Performance

In the unidomain analyses (Fig. 1, top panel) hippocampal radial distance was significantly associated with worse baseline memory performance (left and right $p_{\text {corrected }}<0.001$ ). Bilaterally significant associations were also seen for the language domain (left $p_{\text {corrected }}=$ 
Fig. 2. Unidomain and multidomain maps showing the association of cognitive decline over time and hippocampal radial distance.

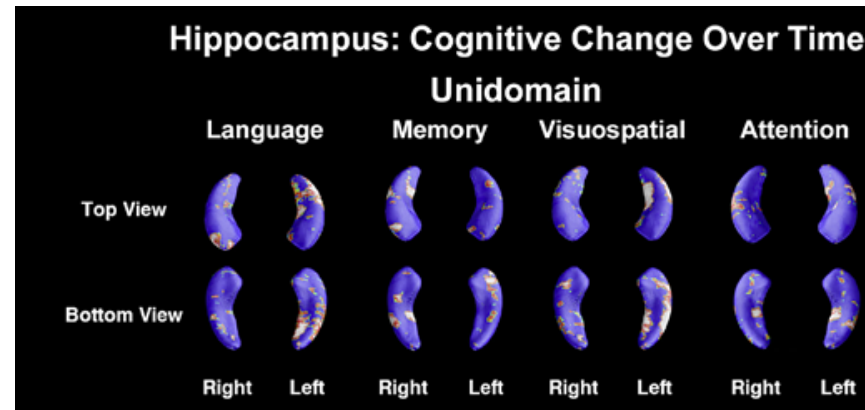

Multidomain Language Memory Visuospatial

Top View

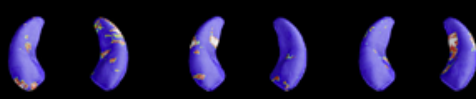

Bottom View
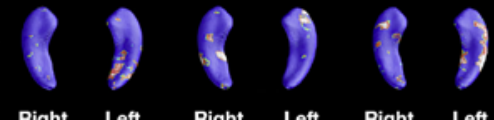

Right

Left

Right Left

Right Left

0.018 , right $p_{\text {corrected }}=0.01$ ). Executive function and attention correlated with hippocampal radial distance on the left only (executive: left $p_{\text {corrected }}=0.001$, right $p_{\text {corrected }}=0.25$; attention: left $p_{\text {corrected }}=0.006$, right $p_{\text {corrected }}=0.22$ ). No significant associations were found in the visuospatial domain (maps not shown).

Based on the unidomain analysis results, four domains - attention, executive, language, and memory - were entered as predictors in the multidomain baseline analysis (Fig. 1, bottom panel). Only memory performance remained significantly associated with hippocampal volume (left $p_{\text {corrected }}<0.001$, right $p_{\text {corrected }}=0.017$ ) while the language domain showed a trend level association on the right (left $p_{\text {corrected }}=0.195$, right $p_{\text {corrected }}=0.098$ ).

\section{Cognitive Change over Time}

In the unidomain analyses (Fig. 2, top panel) a smaller left radial distance was associated with greater decline in language and visuospatial performance (language: left $p_{\text {corrected }}=$ 0.007 , right $p_{\text {corrected }}=0.11$; visuospatial: left $p_{\text {corrected }}=0.001$, right $p_{\text {corrected }}=0.41$ ). Significant right-sided atrophy was predictive of memory decline over time (left $p_{\text {corrected }}=0.4$, right $p_{\text {corrected }}=0.021$ ) and showed a trend-level association with decline in attention (left $p_{\text {corrected }}=$ 0.32 , right $p_{\text {corrected }}=0.086$ ). No significant associations were found for the executive domain (maps not shown).

In the multidomain model (Fig. 2, bottom panel) with language, memory, and visuospatial entered as predictors, memory and visuospatial decline were independently associated with hippocampal atrophy on the right and left, respectively (memory: left $p_{\text {corrected }}=$ 0.35 , right $p_{\text {corrected }}=0.047$; visuospatial: left $p_{\text {corrected }}=0.022$, right $p_{\text {corrected }}=0.56$ ) .

Ventricular Results

Baseline Performance

In the unidomain analyses (Fig. 3, left panel) significant left-sided ventricular enlargement was associated with poor baseline language scores (left $p_{\text {corrected }}=0.041$, right $p_{\text {corrected }}=$ 


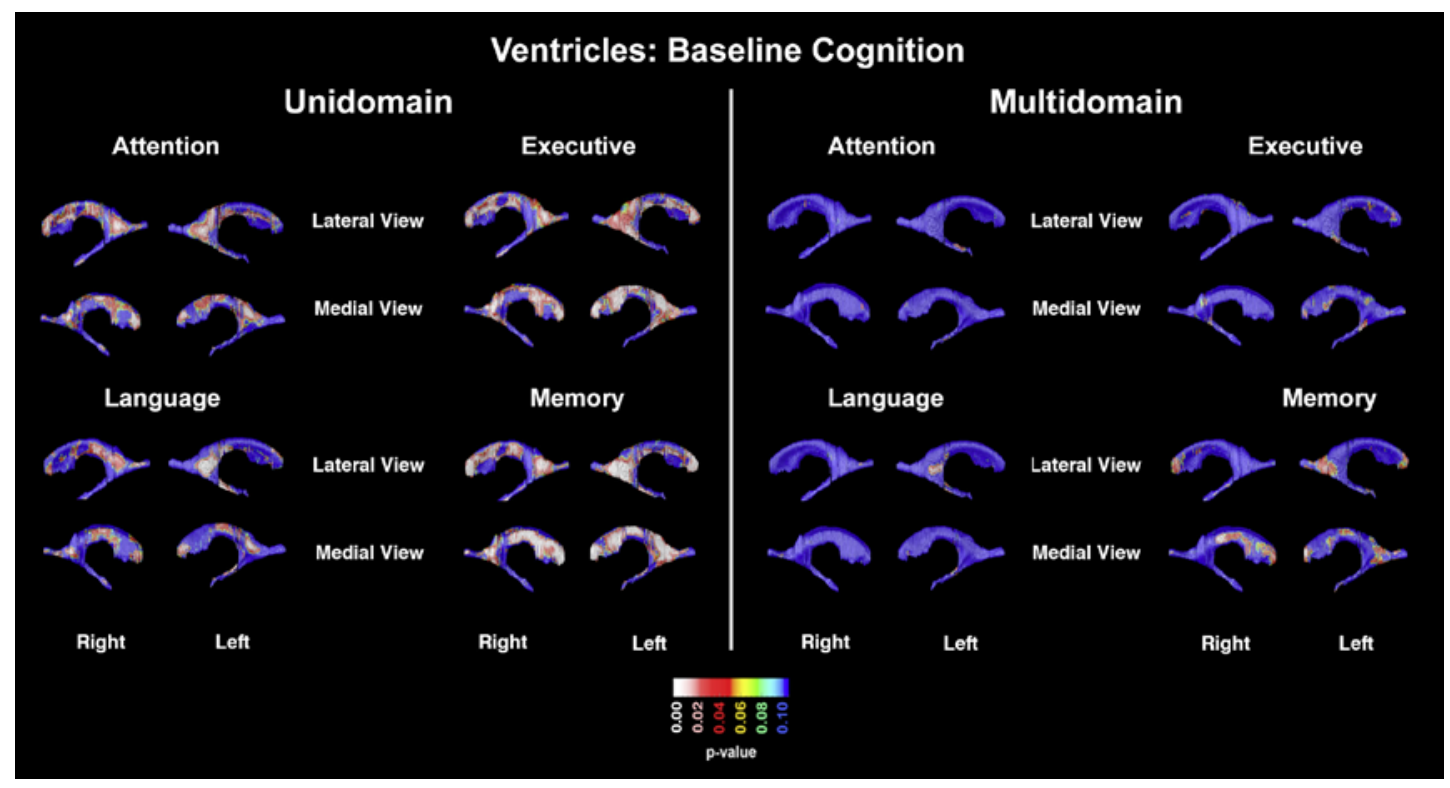

Fig. 3. Unidomain and multidomain maps showing the association of baseline cognitive performance and ventricular radial distance.

0.11). This was largely driven by the left temporal horn $\left(p_{\text {corrected }}=0.016\right)$ while the left occipital and left frontal horns were trending (left occipital $p_{\text {corrected }}=0.052$; left frontal $\left.p_{\text {corrected }}=0.054\right)$. While the right occipital horn was trending $\left(p_{\text {corrected }}=0.052\right)$, this did not survive at the whole ventricle level $\left(p_{\text {corrected }}=0.11\right)$.

Left ventricular enlargement also associated with poor memory performance $\left(p_{\text {corrected }}=\right.$ 0.02 ). This was largely driven by the occipital and frontal horns (left occipital $p_{\text {corrected }}=0.003$; left frontal $p_{\text {corrected }}=0.003$ ). Trend-level association was observed for the left temporal horn $\left(p_{\text {corrected }}=0.054\right)$. Significant associations between ventricular enlargement and memory decline were observed in the right occipital and frontal horns (right occipital $p_{\text {corrected }}=0.024$, right frontal $\left.p_{\text {corrected }}=0.005\right)$; however, these did not survive at the whole ventricle level $\left(p_{\text {corrected }}=0.14\right)$.

Ventricular enlargement showed trend-level associations with baseline performance in the executive domain (left $p_{\text {corrected }}=0.072$ and right $p_{\text {corrected }}=0.063$ ), resulting from significant associations with the frontal and occipital horns (left frontal $p_{\text {corrected }}=0.004$, right frontal $p_{\text {corrected }}=0.011$; left occipital $p_{\text {corrected }}=0.021$, right occipital $p_{\text {corrected }}=$ $0.020)$.

Poor performance in the attention domain showed a trend-level association with right ventriculomegaly (right $p_{\text {corrected }}=0.091$ ) due to significant associations with the right frontal $\left(p_{\text {corrected }}=0.031\right)$ and trend-level effect in the right occipital horn $\left(p_{\text {corrected }}=0.086\right)$. The associations on the left (left occipital horn $p_{\text {corrected }}=0.044$ and left frontal horn $p_{\text {corrected }}=$ 0.053 ) did not survive on the global ventricular level (left $p_{\text {corrected }}=0.103$ ). No significant associations were observed between ventricular enlargement and the visuospatial domain (maps not shown).

Based on the unidomain analysis results, four domains - attention, executive, language, and memory - were entered as predictors in the multidomain baseline analysis (Fig. 3, right panel). None of these domains proved to be significant at the whole ventricle level in the multidomain model. The left temporal horn (left $p_{\text {corrected }}=0.006$ ) was significantly associated with poor executive performance. 


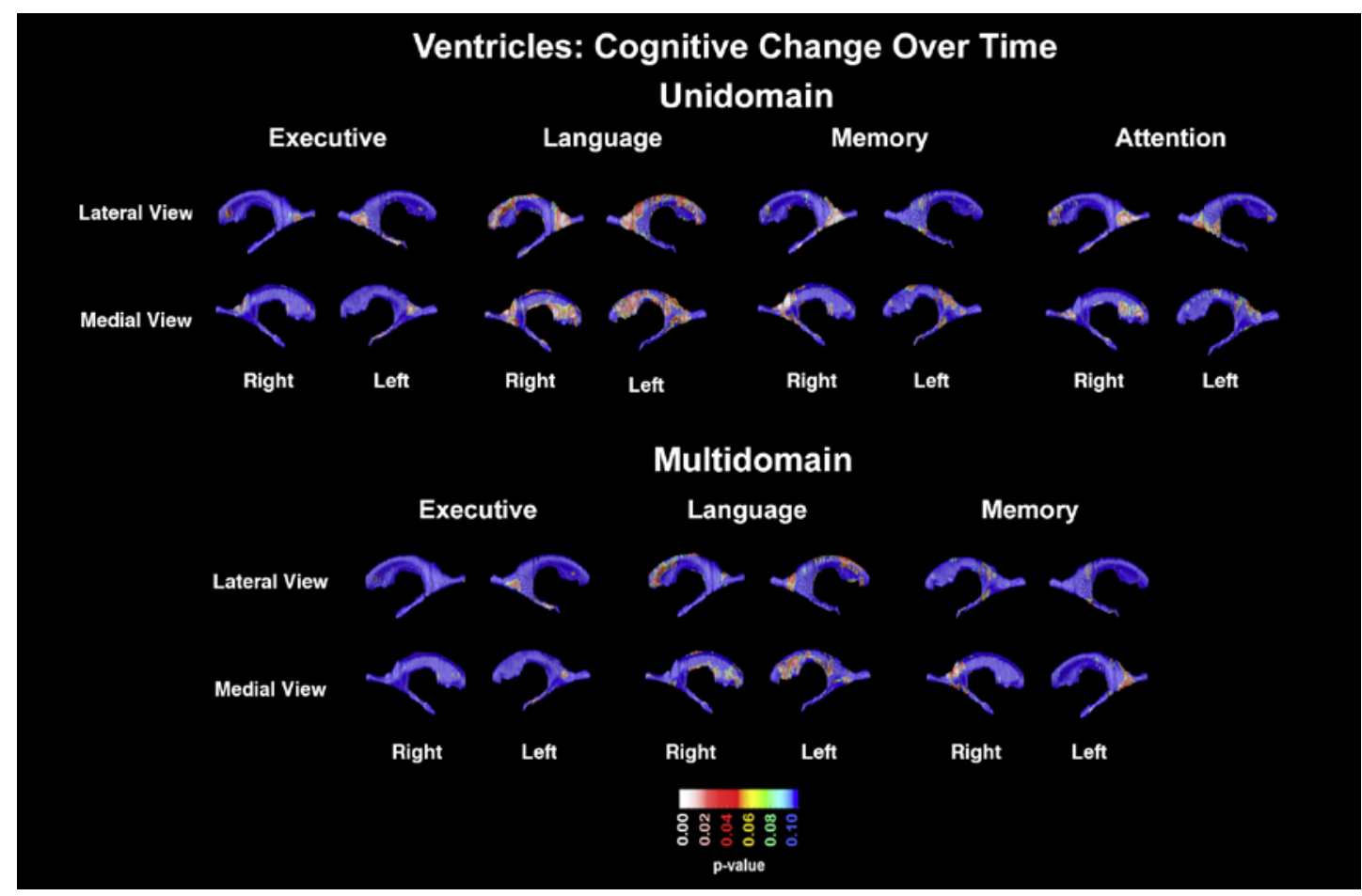

Fig. 4. Unidomain and multidomain maps showing the association of cognitive decline over time and ventricular radial distance.

\section{Cognitive Change over Time}

In our longitudinal analyses (Fig. 4, top panel), we found a trend-level association between right ventricular enlargement and the language domain $\left(p_{\text {corrected }}=0.094\right)$. This effect was driven by the occipital (right $p_{\text {corrected }}=0.02$, left $p_{\text {corrected }}=0.063$ ) and frontal horns (left $p_{\text {corrected }}=0.052$, right $p_{\text {corrected }}=0.06$ ). Right occipital horn enlargement was associated with a decline in memory (right $p_{\text {corrected }}=0.0053$ ) and showed a trend level effect for attention $\left(p_{\text {corrected }}=0.057\right)$. Similar to the baseline analyses, the executive domain was significantly predicted by the left inferior horn $\left(p_{\text {corrected }}=0.037\right)$. The visuospatial domain showed no significant associations (maps not shown).

At the multidomain level with executive, language, and memory entered as predictors, no significant associations were observed across the ventricles as a whole. Ventricular enlargement of the left inferior horn was predictive of decline in the executive domain at the trend level $\left(p_{\text {corrected }}=0.081\right.$; Fig. 4 , bottom panel).

\section{Discussion}

Although nearly all baseline and longitudinal domains were associated with hippocampal radial distance in the unidomain model, only a few survived in the multidomain analyses. Baseline memory performance and memory decline over time were associated with hippocampal atrophy in agreement with the previous literature [28-31,33]. The association at baseline was bilateral, whereas the longitudinal association lateralized to the right. Left-sided hippocampal atrophy predicted longitudinal decline in visuospatial function. Although hippocampal atrophy has been previously associated with worse spatial memory and difficulties with spatial navigation [66, 67], the reason we are observing a left-sided and not right-sided association is not clear. 
Goukasian et al.: Cognitive Correlates of Hippocampal Atrophy and Ventricular Enlargement

As opposed to the hippocampus, the lateral ventricles are large structures that traverse the full length of the hemispheres and are embedded in all lobes. In the context of our analyses, we felt that for such large structures it is important to not only look at global effects, but also at regional effect on the specific horns of the lateral ventricles. Dilation of individual horns corresponds to lobar atrophy of the brain and can be differentially associated with different cognitive function. In addition to associations with overall global ventriculomegaly, we have also found the associations with the frontal, temporal, and occipital horns. While all domains except the visuospatial showed major ventricular associations with the frontal and occipital horns in the unidomain analyses, none survived in the multidomain analyses. These findings suggest that the lateral ventricles might be an imaging biomarker more closely related to global cognitive decline rather than to specific cognitive functions. This is further supported by several of our previous publications using the same technique with different study cohorts with either Parkinson's disease or AD [7, 68, 69].

There are several strengths and limitations of our study that should be considered. The greatest strength of this study is the advanced imaging techniques that significantly reduce the signal-to-noise ratio by explicitly matching corresponding points on the ventricular and hippocampal surfaces. By virtue of this, we achieved a much higher statistical power to detect focal disease-associated effects and associations. Though we use the term "atrophy" for our analyses here, we are reporting cross-sectional ventricular and hippocampal analyses only. Given the original scope of our study we also only focused on NC and MCI subjects with MMSE scores $>24$, limiting our results to the early stages of neurodegeneration and, thus, our findings can only be generalized to the early disease stages.

\section{Acknowledgments}

The authors would like to thank all of the research participants who were willing to participate in this study, as well as the clinicians and staff members for their dedicated time and hard work in collecting data to make this study possible.

\section{Statement of Ethics}

Informed consent was administered and obtained according to the guidelines of the UCLA Institutional Review Board.

\section{Disclosure Statement}

Liana G. Apostolova received research support from General Electric Healthcare and Araclon Biotech, and served on the speaker's bureau for Eli Lilly and Company and Piramal Enterprises and on the advisory board for Eli Lilly and Company. All other authors have no disclosures.

\section{Funding Sources}

This work was generously supported by NIA R01 AG040770, NIA K02 AG048240, NIA P50 AG16570, NIA P30 AG010133, NIA U01AG024904 and the Easton Consortium for Alzheimer's Drug Discovery and Biomarker Development. 
Goukasian et al.: Cognitive Correlates of Hippocampal Atrophy and Ventricular Enlargement

\section{References}

1 Hebert LE, Weuve J, Scherr PA, Evans DA. Alzheimer disease in the United States (2010-2050) estimated using the 2010 census. Neurology. 2013;80(19):1778-83.

2 Alzheimer's A; Alzheimer's Association. 2016 Alzheimer's disease facts and figures. Alzheimers Dement. 2016 Apr;12(4):459-509.

3 Gauthier S, Reisberg B, Zaudig M, Petersen RC, Ritchie K, Broich K, et al.; International Psychogeriatric Association Expert Conference on mild cognitive impairment. Mild cognitive impairment. Lancet. 2006 Apr; 367(9518):1262-70.

4 Petersen RC. Mild cognitive impairment as a diagnostic entity. J Intern Med. 2004 Sep;256(3):183-94.

5 Petersen RC, Doody R, Kurz A, Mohs RC, Morris JC, Rabins PV, et al. Current concepts in mild cognitive impairment. Arch Neurol. 2001 Dec;58(12):1985-92.

6 Petersen RC, Smith GE, Waring SC, Ivnik RJ, Tangalos EG, Kokmen E. Mild cognitive impairment: clinical characterization and outcome. Arch Neurol. 1999 Mar;56(3):303-8.

7 Apostolova LG, Green AE, Babakchanian S, Hwang KS, Chou YY, Toga AW, et al. Hippocampal atrophy and ventricular enlargement in normal aging, mild cognitive impairment (MCI), and Alzheimer Disease. Alzheimer Dis Assoc Disord. 2012;26(1):17-27.

8 Carmichael OT, Kuller LH, Lopez OL, Thompson PM, Dutton RA, Lu A, et al. Cerebral ventricular changes associated with transitions between normal cognitive function, mild cognitive impairment, and dementia. Alzheimer Dis Assoc Disord. 2007;21(1):14-24.

9 Driscoll I, Davatzikos C, An Y, Wu X, Shen D, Kraut M, et al. Longitudinal pattern of regional brain volume change differentiates normal aging from MCI. Neurology. 2009;72(22):1906-13.

10 Thompson PM, Hayashi KM, De Zubicaray GI, Janke AL, Rose SE, Semple J, et al. Mapping hippocampal and ventricular change in Alzheimer disease. Neuroimage. 2004 Aug;22(4):1754-66.

11 Apostolova LG, Dutton RA, Dinov ID, Hayashi KM, Toga AW, Cummings JL, et al. Conversion of mild cognitive impairment to Alzheimer disease predicted by hippocampal atrophy maps. Arch Neurol. 2006 May; 63(5): 693-9.

12 Apostolova LG, Mosconi L, Thompson PM, Green AE, Hwang KS, Ramirez A, et al. Subregional hippocampal atrophy predicts Alzheimer's dementia in the cognitively normal. Neurobiol Aging. 2010;31(7):1077-88.

13 Apostolova LG, Zarow C, Biado K, Hurtz S, Boccardi M, Somme J, et al. Relationship between hippocampal atrophy and neuropathology markers: a 7T MRI validation study of the EADC-ADNI Harmonized Hippocampal Segmentation Protocol. Alzheimers Dement. 2015;11(2):139-50.

14 Blanken AE, Hurtz S, Zarow C, Biado K, Honarpisheh H, Somme J, et al. Associations between hippocampal morphometry and neuropathologic markers of Alzheimer's disease using 7 T MRI. Neuroimage Clin. 2017;15: 56-61.

15 Nestor SM, Rupsingh R, Borrie M, Smith M, Accomazzi V, Wells JL, et al. Ventricular enlargement as a possible measure of Alzheimer's disease progression validated using the Alzheimer's disease neuroimaging initiative database. Brain. 2008;131(Pt 9):2443-54.

16 Bradley KM, Bydder GM, Budge MM, Hajnal JV, White SJ, Ripley BD, et al. Serial brain MRI at 3-6 month intervals as a surrogate marker for Alzheimer's disease. Br J Radiol. 2002 Jun;75(894):506-13.

17 Burns A, Jacoby R, Levy R. Computed tomography in Alzheimer's disease: a longitudinal study. Biol Psychiatry. $1991 \mathrm{Feb} ; 29(4): 383-90$.

18 de Leon MJ, Golomb J, George AE, Convit A, Tarshish CY, McRae T, et al. The radiologic prediction of Alzheimer disease: the atrophic hippocampal formation. AJNR Am J Neuroradiol. 1993 Jul-Aug;14(4):897-906.

19 DeCarli C, Haxby JV, Gillette JA, Teichberg D, Rapoport SI, Schapiro MB. Longitudinal changes in lateral ventricular volume in patients with dementia of the Alzheimer type. Neurology. 1992 Oct;42(10):2029-36.

20 Luxenberg JS, Haxby JV, Creasey H, Sundaram M, Rapoport SI. Rate of ventricular enlargement in dementia of the Alzheimer type correlates with rate of neuropsychological deterioration. Neurology. 1987 Jul;37(7):113540.

21 Shear PK, Sullivan EV, Mathalon DH, Lim KO, Davis LF, Yesavage JA, et al. Longitudinal volumetric computed tomographic analysis of regional brain changes in normal aging and Alzheimer's disease. Arch Neurol. 1995 Apr;52(4):392-402.

22 Apostolova LG, Babakchanian S, Hwang KS, Green AE, Zlatev D, Chou YY, et al. Ventricular enlargement and its clinical correlates in the imaging cohort from the ADCS MCI donepezil/vitamin E study. Alzheimer Dis Assoc Disord. 2013;27(2):174-81.

23 Apostolova LG, Thompson PM. Brain mapping as a tool to study neurodegeneration. Neurotherapeutics. 2007; $4(3): 387-400$.

24 Evans MC, Barnes J, Nielsen C, Kim LG, Clegg SL, Blair M, et al.; Alzheimer's Disease Neuroimaging Initiative. Volume changes in Alzheimer's disease and mild cognitive impairment: cognitive associations. Eur Radiol. 2010 Mar;20(3):674-82.

25 Ferrarini L, Palm WM, Olofsen H, van der Landen R, Jan Blauw G, Westendorp RG, et al. MMSE scores correlate with local ventricular enlargement in the spectrum from cognitively normal to Alzheimer disease. Neuroimage. 2008 Feb;39(4):1832-8.

26 Iachini I, Iavarone A, Senese VP, Ruotolo F, Ruggiero G. Visuospatial memory in healthy elderly, AD and MCI: a review. Curr Aging Sci. 2009 Mar;2(1):43-59. 
27 Nagata T, Shinagawa S, Ochiai Y, Aoki R, Kasahara H, Nukariya K, et al. Association between executive dysfunction and hippocampal volume in Alzheimer's disease. Int Psychogeriatr. 2011 Jun;23(5):764-71.

28 Peng GP, Feng Z, He FP, Chen ZQ, Liu XY, Liu P, et al. Correlation of hippocampal volume and cognitive performances in patients with either mild cognitive impairment or Alzheimer's disease. CNS Neurosci Ther. 2015 Jan;21(1):15-22.

29 Petersen RC, Jack CR Jr, Xu YC, Waring SC, O'Brien PC, Smith GE, et al. Memory and MRI-based hippocampal volumes in aging and AD. Neurology. 2000 Feb;54(3):581-7.

30 Scheltens P, Leys D, Barkhof F, Huglo D, Weinstein HC, Vermersch P, et al. Atrophy of medial temporal lobes on MRI in "probable" Alzheimer's disease and normal ageing: diagnostic value and neuropsychological correlates. J Neurol Neurosurg Psychiatry. 1992;55(10):967-72.

31 Teipel SJ, Ewers M, Wolf S, Jessen F, Kolsch H, Arlt S, et al. Multicentre variability of MRI-based medial temporal lobe volumetry in Alzheimer's disease. Psychiatry Res. 2010 Jun;182(3):244-50.

32 Visser PJ, Scheltens P, Verhey FR, Schmand B, Launer LJ, Jolles J, et al. Medial temporal lobe atrophy and memory dysfunction as predictors for dementia in subjects with mild cognitive impairment. J Neurol. 1999 Jun;246(6):477-85.

33 Vyhnalek M, Nikolai T, Andel R, Nedelska Z, Rubinova E, Markova H, et al. Neuropsychological correlates of hippocampal atrophy in memory testing in nondemented older adults. J Alzheimers Dis. 2014;42(suppl 3):S81-90.

34 Thompson PM, Apostolova LG. Computational anatomical methods as applied to ageing and dementia. Br J Radiol. 2007 Dec;80(Spec No 2):S78-91.

35 McKhann G, Drachman D, Folstein M, Katzman R, Price D, Stadlan EM. Clinical diagnosis of Alzheimer's disease: report of the NINCDS-ADRDA Work Group under the auspices of Department of Health and Human Services Task Force on Alzheimer's Disease. Neurology. 1984 Jul;34(7):939-44.

36 Folstein MF, Folstein SE, McHugh PR. "Mini-mental state". A practical method for grading the cognitive state of patients for the clinician. J Psychiatr Res. 1975 Nov;12(3):189-98.

37 Wechsler D. Wechsler Test of Adult Reading. San Antonio: TX Psychological Corporation; 2001.

38 Wechsler D. Manual for the Wechsler Adult Intelligence Scale. 3rd ed. San Antonio: TX Psychological Corporation; 1997.

39 Wechsler D. Wechsler Memory Scale. Third Edition manual. San Antonio: TX Psychological Corporation; 1997.

40 Reitan RM, Wolfson D. The Halstead-Reitan Neuropsychological Test Battery: Therapy and Clinical Interpretation. Tucson (AZ): Neuropsychological Press; 1985.

41 Stroop JR. Studies in interference of serial verbal reactions. J Exp Psychol. 1935;18(6):643-62.

42 Kaplan EF, Goodgalss H, Weintraub S. The Boston naming test. Philadelphia: Lea and Febiger; 1983.

43 Benton AL, Hamsher K. Multilingual apahasia examination. Iowa City (Iowa): AJA Associates; 1989.

44 Osterrieth PA. Le test de copie d'une figure complexe. Arch Psychol. 1944;30:206-356.

45 Delis DC, Kramer JH, Kaplan E, Ober BA. California Verbal Learning Test, Form II. 2nd ed. San Antonio (TX): The Psychological Corporation; 1987.

46 Heaton RK, Chelune GJ, Talley JL, Kay GG, Curtiss G. Wisconsin Card Sorting Test Manual: Revised and expanded. Odessa (FL): Psychological Assessment Resources Inc; 1993.

47 Wilhalme H, Goukasian N, De Leon F, He A, Hwang KS, Woo E, et al. A comparison of theoretical and statistically derived indices for predicting cognitive decline. Alzheimers Dement. 2017;6:171-81.

48 Mazziotta J, Toga A, Evans A, Fox P, Lancaster J, Zilles K, et al. A probabilistic atlas and reference system for the human brain: International Consortium for Brain Mapping (ICBM). Philos Trans R Soc Lond B Biol Sci. 2001 Aug;356(1412):1293-322.

49 Collins DL, Neelin P, Peters TM, Evans AC. Automatic 3D intersubject registration of MR volumetric data in standardized Talairach space. J Comput Assist Tomogr. 1994 Mar-Apr;18(2):192-205.

50 Shattuck DW, Leahy RM. BrainSuite: an automated cortical surface identification tool. Med Image Anal. 2002 Jun;6(2):129-42.

51 Shattuck DW, Sandor-Leahy SR, Schaper KA, Rottenberg DA, Leahy RM. Magnetic resonance image tissue classification using a partial volume model. Neuroimage. 2001 May;13(5):856-76.

52 Boccardi M, Bocchetta M, Apostolova LG, Barnes J, Bartzokis G, Corbetta G, et al. Delphi definition of the EADCADNI Harmonized Protocol for hippocampal segmentation on magnetic resonance. Alzheimers Dement. 2015; 11(2):126-38.

53 Bocchetta M, Boccardi M, Ganzola R, Apostolova LG, Preboske G, Wolf D, et al. Harmonized benchmark labels of the hippocampus on magnetic resonance: the EADC-ADNI project. Alzheimers Dement. 2015;11(2):151-60. e5.

54 Frisoni GB, Jack CR Jr, Bocchetta M, Bauer C, Frederiksen KS, Liu Y, et al. The EADC-ADNI Harmonized Protocol for manual hippocampal segmentation on magnetic resonance: evidence of validity. Alzheimers Dement. 2015;11(2):111-25.

55 Duvernoy HM. The human hippocampus: an atlas of applied anatomy. Berlin: Springer; 1988.

56 Mai JK, Paxinos G, Assheuer JK. Atlas of the Human Brain. San Diego (CA): Elsevier Academic Press; 2004.

57 Apostolova LG, Morra JH, Green AE, Hwang KS, Avedissian C, Woo E, et al. Automated 3D mapping of baseline and 12-month associations between three verbal memory measures and hippocampal atrophy in $490 \mathrm{ADNI}$ subjects. Neuroimage. 2010;51(1):488-99. 
Goukasian et al.: Cognitive Correlates of Hippocampal Atrophy and Ventricular Enlargement

58 Freund Y, Schapire RE. A decision-theoretic generalization of on-line learning and an application to boosting. J Comput Syst Sci. 1997;55(1):119-39.

59 Morra JH, Tu Z, Apostolova LG, Green AE, Avedissian C, Madsen SK, et al. Mapping hippocampal degeneration in 400 subjects with a novel automated segmentation approach. 5th IEEE International Symposium on Biomedical Imaging: From Nano to Macro, ISBI - Paris; 2008. p. 336-9.

60 Morra JH, Tu Z, Apostolova LG, Green AE, Avedissian C, Madsen SK, et al. Validation of a fully automated 3D hippocampal segmentation method using subjects with Alzheimer's disease mild cognitive impairment, and elderly controls. Neuroimage. 2008;43(1):59-68.

61 Morra JH, Tu Z, Apostolova LG, Green AE, Avedissian C, Madsen SK, et al. Automated mapping of hippocampal atrophy in 1-year repeat MRI data from 490 subjects with Alzheimer's disease, mild cognitive impairment, and elderly controls. Neuroimage. 2009;45(1 Suppl):S3-15.

62 Chou YY, Lepore N, de Zubicaray GI, Carmichael OT, Becker JT, Toga AW, et al. Automated ventricular mapping with multi-atlas fluid image alignment reveals genetic effects in Alzheimer's disease. Neuroimage. 2008; 40(2):615-30.

63 Apostolova LG, Dinov ID, Dutton RA, Hayashi KM, Toga AW, Cummings JL, et al. 3D comparison of hippocampal atrophy in amnestic mild cognitive impairment and Alzheimer's disease. Brain. 2006 Nov;129(Pt 11):286773.

64 Apostolova LG, Steiner CA, Akopyan GG, Dutton RA, Hayashi KM, Toga AW, et al. Three-dimensional gray matter atrophy mapping in mild cognitive impairment and mild Alzheimer disease. Arch Neurol. 2007;64(10): 1489-95.

65 Apostolova LG, Thompson PM, Green AE, Hwang KS, Zoumalan C, Jack CR Jr, et al. 3D comparison of low, intermediate, and advanced hippocampal atrophy in MCI. Hum Brain Mapp. 2010;31(5):786-97.

66 Beudel M, Leenders KL, de Jong BM. Hippocampus activation related to 'real-time' processing of visuospatial change. Brain Res. 2016 Dec;1652:204-11.

67 Laczó J, Andel R, Nedelska Z, Vyhnalek M, Vlcek K, Crutch S, et al. Exploring the contribution of spatial navigation to cognitive functioning in older adults. Neurobiol Aging. 2017 Mar;51:67-70.

68 Apostolova L, Alves G, Hwang KS, Babakchanian S, Bronnick KS, Larsen JP, et al. Hippocampal and ventricular changes in Parkinson's disease mild cognitive impairment. Neurobiol Aging. 2012;33(9):2113-24.

69 Apostolova LG, Beyer M, Green AE, Hwang KS, Morra JH, Chou YY, et al. Hippocampal, caudate, and ventricular changes in Parkinson's disease with and without dementia. Mov Disord. 2010;25(6):687-95. 